\title{
Análise da densidade óptica da sutura palatina mediana seis meses após a expansão rápida da maxila assistida cirurgicamente
}

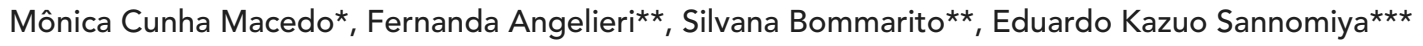

\section{Resumo}

Objetivo: avaliar a neoformação óssea da sutura palatina mediana por meio da análise de densidade óptica após a expansão rápida da maxila assistida cirurgicamente (ERMAC). Métodos: a amostra estudada consistiu de 16 pacientes, sendo 6 do gênero masculino e 10 do gênero feminino. Todos os pacientes apresentavam atresia maxilar com mordida cruzada posterior e foram submetidos à ERMAC. Foram realizadas radiografias oclusais parciais da região dos incisivos superiores em quatro fases: antes da ERMAC (fase I), após o fim da ativação do parafuso expansor (fase II), três meses após a ativação do parafuso expansor (fase III), e seis meses após a ERMAC (fase IV). Uma escala de alumínio com oito degraus, variando de 1 a $8 \mathrm{~mm}$, foi adaptada no extremo das radiografias. A análise da densidade óptica foi feita após a digitalização das radiografias, utilizando-se o software Image Too ${ }^{\circledR}$ (UTHSCSA, San Antonio, Texas, EUA). Em cada uma dessas fases, duas áreas foram selecionadas e analisadas. A região "A" (anterior) mediu 8 x $1 \mathrm{~mm}^{2}$ e localizou-se a $1,2 \mathrm{~cm}$ da tangente situada nos incisivos centrais superiores na região da sutura palatina mediana. A região "B" (posterior) mediu $5 \times 9 \mathrm{~mm}^{2}$ e localizou-se a 4,3cm da tangente situada nos incisivos centrais superiores na região da sutura palatina mediana. Resultados: na região "A", comparandose a fase I com a fase II, e a fase I com a fase III, foram encontradas diferenças estatisticamente significativas $-\mathrm{p}<0,0001$ e $\mathrm{p}<0,0001$, respectivamente -; comparando-se a fase I com a IV, e a fase II com a III, foram encontradas diferenças estatisticamente significativas $-p=0,0169$ e $\mathrm{p}=0,0004$, respectivamente -; comparando-se as fases II e IV, e a fase III com a IV, diferenças estatisticamente significativas também foram encontradas $-p<0,0001$ e $p=0,0023$, respectivamente. Na região "B", comparando-se as fases I e II, e a fase I com a III, diferenças estatisticamente significativas foram encontradas $-\mathrm{p}<0,0001$ e $\mathrm{p}<0,0001$, respectivamente -; comparando-se a fase I com a fase IV, nenhuma diferença estatisticamente significativa foi observada $(p=0,1152)$; comparando-se a fase II com a fase III, a fase II com a IV, e a fase III com a IV, diferenças estatisticamente significativas foram encontradas $-p=0,0002, p<0,0001$ e $p=0,0010$, respectivamente. Conclusões: a análise da densidade óptica permite ao cirurgião bucomaxilofacial e ao ortodontista um controle melhor do tratamento após a ERMAC. Seis meses após a ERMAC, a neoformação óssea da sutura palatina mediana não está completa na região "A" $(\mathrm{p}<0,05)$, mas na região "B" nenhuma diferença estatisticamente significativa foi observada $(p>0,05)$ entre as fases I e IV.

Palavras-chave: Radiografia. Densidade óptica. Neoformação óssea. Expansão rápida da maxila.

* Mestre em Ortodontia pela Universidade Metodista de São Paulo (Umesp).

** Professoras doutoras do programa de pós-graduação em Odontologia, área de concentração em Ortodontia, da Umesp.

*** Professor doutor do Programa de pós-graduação em Odontologia, área de concentração Ortodontia, da Umesp. Professor titular da disciplina de Imaginologia Bucomaxilofacial da Faculdade de Odontologia da Umesp. 


\section{INTRODUÇÃO E REVISÃO DE LITERATURA}

A atresia ou deficiência transversal da maxila é uma deficiência dentofacial caracterizada pela presença de: mordida cruzada posterior uni ou bilateral, palato profundo ou ogival, apinhamento dentário e respiração nasal deficiente. A expansão rápida da maxila assistida cirurgicamente (ERMAC) é um procedimento clínico terapêutico usado para corrigir a deficiência transversal da maxila. Em casos de adultos, que já tenham completado a ossificação da sutura palatina mediana, o tratamento envolve a ERMAC, a fim de permitir sua separação por meio de aparelhos ortodônticos devidamente confeccionados e adaptados para esse propósito².

A sutura palatina mediana aparece nas radiografias oclusais como uma linha estreita radiolúcida. Imediatamente após a expansão rápida maxilar, a linha radiolúcida da sutura torna-se mais larga. Cobo, Vijande e Quintanilla ${ }^{4}$ enfatizaram que estudos da maxila e da sutura palatina mediana durante a expansão rápida da maxila e suas alterações são importantes para os ortodontistas, a fim de que esses possam prever os efeitos do tratamento ortopédico. A análise dessa neoformação óssea por meio da avaliação da densidade óptica de radiografias oclusais digitalizadas, bem como o uso de uma escala de alumínio, constituem um método para o controle do tratamento pós-operatório. Com isso, é possível quantificar a neoformação óssea e determinar a área de referência a ser analisada para que se possa controlar o processo de ossificação.

Vários estudos ${ }^{4,7,14-17}$ foram relatados, na literatura, utilizando a análise da densidade óptica.

$\mathrm{Melo}^{7}$ mensurou as densidades ópticas da sutura palatina mediana antes da expansão da maxila, imediatamente após essa expansão e após três meses de contenção. A amostra foi constituída por 31 pacientes que se submeterem à expansão rápida da maxila, os quais se encontravam em fase de crescimento, e foi dividida em: grupo I - constituído pelos pacientes de dentadura mista; e grupo II - com os pacientes de dentadura permanente. Foram obtidas radiografias oclusais e as imagens foram digitalizadas por meio de um sistema de radiografias digitais por placa fotoativada. Áreas de interesse foram demarcadas nas radiografias digitalizadas, procedendo-se à leitura das densidades ópticas pelo programa Digora for Windows 2.1 ${ }^{\circledR}$ (Soredex, Finlândia). A autora concluiu que: as densidades ópticas da sutura palatina mediana na fase final mostraram valores inferiores aos da fase inicial nos pacientes com dentadura mista; as densidades ópticas da sutura palatina mediana na fase final mostraram valores semelhantes aos da fase inicial nos pacientes com dentadura permanente; a densidade óptica final da sutura palatina mediana não corresponde à inicial nos pacientes do gênero feminino do grupo de dentadura mista; a densidade óptica final da sutura palatina mediana é semelhante à inicial nos pacientes do gênero masculino do grupo de dentadura mista; para os pacientes do grupo de dentadura permanente, houve equivalência nos valores de densidades ópticas inicial e final, tanto para os pacientes do gênero masculino como os do feminino.

Simões et al. ${ }^{15}$ avaliaram a neoformação óssea utilizando a análise da densidade óptica na região da sutura palatina mediana após a expansão da maxila. Para tanto, foram analisadas 109 radiografias, de 37 pacientes, nos estágios pré-expansão e nos três primeiros meses de contenção. As radiografias foram digitalizadas com o auxílio de um scanner e foi utilizado o programa DentScan Dentview $^{\circledR}$ (Apica, Israel) para proceder as leituras. Três áreas ao longo da sutura palatina mediana, uma na região anterior e duas na posterior, foram determinadas para a realização das medidas. De acordo com os resultados, os autores concluiu que a densidade óptica na região da sutura palatina mediana nas diferentes fases da terapia expansionista apresentou grande variação, compatível com a abertura da referida sutura e posterior neoformação óssea nos meses em contenção. 
Observou-se valores crescentes para as densidades ópticas médias nos períodos em contenção. Isso sugeriu neoformação óssea na região da sutura palatina mediana. Nas três áreas estudadas, foi observada uma diminuição da densidade óptica pré-tratamento, quando comparada à do primeiro mês em contenção. Do primeiro para o segundo mês, verificou-se um aumento na mesma. Houve comportamento semelhante entre o segundo e o terceiro mês em contenção. Entretanto, o valor da densidade óptica obtido no terceiro mês não foi equivalente ao valor pré-tratamento. Vasconcelos et al. ${ }^{17}$ avaliaram a remodelação óssea na região da sutura palatina mediana por meio da densidade óptica em radiografias oclusais digitalizadas, obtidas periodicamente, de pacientes adultos submetidos à ERMAC. A amostra constou de 126 radiografias oclusais convencionais obtidas nas fases pré-expansão, pós-expansão imediata e contenção de até 120 dias, as quais foram digitalizadas por meio de um scanner ScanMaker 9800 XL (Microtec International, Taiwan) com leitor de transparências e analisadas no programa editor de imagens Adobe Photoshop (Adobe, EUA) para a obtenção dos valores de pixels correspondentes à densidade óptica na região da sutura. Após a análise estatística, foi constatado que os valores finais da densidade óptica foram significativamente inferiores em relação aos valores iniciais, no período de 120 dias de contenção fixa. Nesses estudos, os autores concordaram que a análise da maturidade óssea por meio da densidade óptica é um método para que os cirurgiões e os ortodontistas controlem os resultados após a expansão ortopédica e cirúrgica da maxila.

\section{PROPOSIÇÃO}

O objetivo da presente pesquisa é avaliar a neoformação óssea da sutura palatina mediana por meio da análise de densidade óptica antes da ERMAC, após o fechamento do parafuso expansor, três meses após o fechamento do parafuso expansor e seis meses após a ERMAC.

\section{MATERIAL E MÉTODOS}

A amostra para esse estudo constituiu-se de 64 radiografias oclusais de 16 pacientes, sendo 6 do gênero masculino e 10 do gênero feminino, com idades variando entre 20 e 45 anos, atendidos por ortodontistas do Curso de Pós-graduação em Ortodontia da Universidade Metodista de São Paulo. Todos os pacientes apresentavam atresia maxilar com mordida cruzada posterior e foram submetidos à ERMAC. O estudo foi aprovado pelo Comitê de Ética em Pesquisas da Universidade Metodista com protocolo $n^{\circ} 67151 / 05$. Os pacientes foram informados a respeito do uso de seus dados para estudos científicos e assinaram uma autorização para os procedimentos cirúrgicos, bem como para o uso desses dados. A ERMAC convencional incluiu a osteotomia do tipo Le Fort I, exceto por não abordar a sutura pterigopalatina, e é associada à osteotomia sagital mediana da maxila.

Os pacientes foram submetidos às radiografias oclusais da maxila nas quatro fases do tratamento. Utilizou-se um aparelho de raios $\mathrm{X}$ modelo Spectro II (Dabi Atlante, Ribeirão Preto/SP), com especificações fixas de 70kvp, 10mA e tempo de exposição de 1 segundo. As radiografias oclusais foram realizadas utilizando-se filme oclusal com medidas de 51 x $71 \mathrm{~mm}$ (Insight film, Eastman Kodak, Rochester, NY, EUA) e orientadas com o uso de um posicionador (Rinn, Elgin, Illinois, EUA), com o intuito de padronizar a obtenção das mesmas. Uma escala de alumínio com oito graus, variando de 1 a $8 \mathrm{~mm}$, foi adaptada no extremo das radiografias para a posterior análise da densidade óptica. A primeira radiografia oclusal foi realizada antes da cirurgia (fase I) e da instalação do expansor Hyrax, que foi utilizado durante o tratamento. O Hyrax é constituído por um parafuso expansor soldado às quatro bandas que são adaptadas aos dentes de suporte. Após a ERMAC, e a cirurgia propriamente dita, o expansor Hyrax foi ativado até a obtenção da abertura desejada. O protocolo de ativação foi o de 1/4 de volta pela manhã e 1/4 de volta à noite, totalizando $2 / 4$ de volta ao dia, 
até quase se obter a mordida de Brodie (cúspide palatina do molar superior com a cúspide vestibular do molar inferior). Depois de finalizada a ativação do parafuso expansor (fechamento - fase II), foi realizada a segunda radiografia oclusal. A terceira radiografia oclusal foi realizada três meses após a suspensão da ativação do parafuso expansor (fase III). A última radiografia foi feita seis meses após a ERMAC (fase IV). Todas as radiografias foram processadas manualmente, com método tempo/temperatura $\left(20 \mathrm{seg} / 30^{\circ} \mathrm{C}\right)$.

Todas as 64 radiografias foram digitalizadas utilizando-se um scanner modelo Power Look 1000 (Umax, Taiwan, China) e um computador Dell Dimension E510 ${ }^{\circledast}$ (Dell, EUA), sendo gravadas em formato tiff, com resolução de 300dpi.

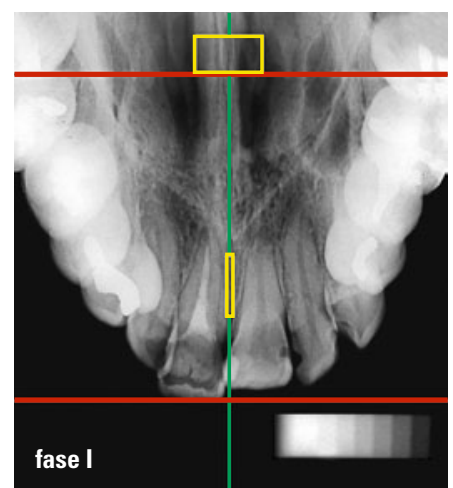

FIGURA 1 - Radiografia oclusal antes da ERMAC.

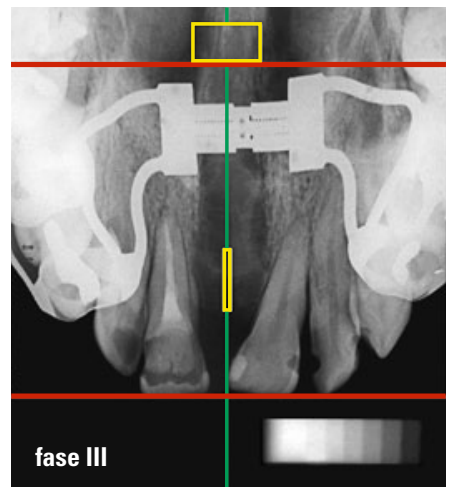

FIGURA 3 - Radiografia oclusal três meses após o fechamento do parafuso expansor.
Cada uma das radiografias foi analisada por meio do histograma e comparada com a escala de alumínio, usando-se o software Image Tool ${ }^{\circledR}$ (UTHSCSA, San Antonio, Texas, EUA).

Em relação à padronização, foram realizadas marcações de áreas e planos na radiografia oclusal digitalizada a ser analisada. Duas regiões da radiografia oclusal foram selecionadas para o estudo. A região "A" mediu $8 \times 1 \mathrm{~mm}^{2}$ e localizou-se a $1,2 \mathrm{~cm}$ da tangente situada nos incisivos centrais superiores na região da sutura palatina mediana. A região "B" mediu 5 x $9 \mathrm{~mm}^{2}$ e localizou-se a $4,3 \mathrm{~cm}$ da tangente situada nos incisivos centrais superiores na região da sutura palatina mediana. Em todas as fases (I, II, III e IV) do estudo foi utilizada essa padronização (Fig. 1 - 4).

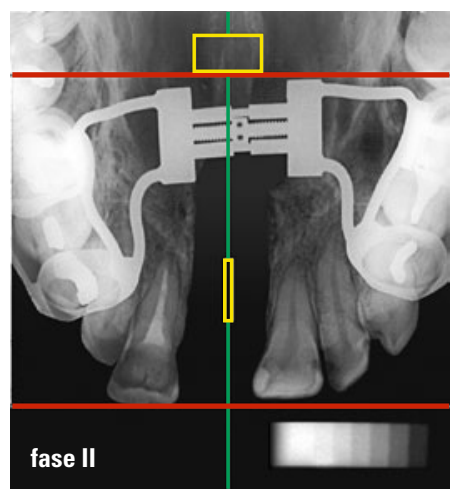

FIGURA 2 - Radiografia oclusal após o fechamento do parafuso expansor.

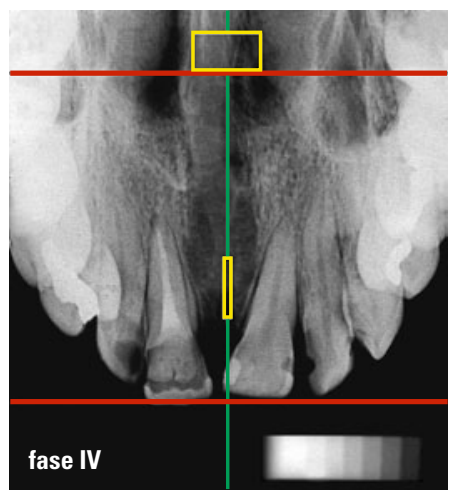

FIGURA 4 - Radiografia oclusal seis meses após a ERMAC. 
Para se alcançar o objetivo desse estudo, as análises estatísticas utilizadas foram as de ANOVA e as comparações múltiplas de Bonferroni, para se avaliar em qual fase do estudo a densidade óptica sofreu alteração ${ }^{9}$. Foram realizados testes com um nível de significância de 5\%.

\section{RESULTADOS}

A amostra toda foi mensurada duas vezes, para o estudo do erro do método. Para calcular o erro casual do método foi utilizada a fórmula proposta por Dahlberg, e para avaliar o erro sistemático intraexaminador foram construídos gráficos de Blend-Altman ${ }^{1}$. A tabela 1 apresenta os erros de medição em cada fase e em cada região, calculados pela fórmula de Dahlberg. Nota-se que o erro casual só ocorreu a partir da terceira casa decimal (Tab. 1), sendo considerado um erro pequeno,

TABELA 1 - Erros casuais calculados pela fórmula de Dahlberg.

$\begin{array}{cccc}\text { região } & \text { fase } & \begin{array}{c}\text { erro casual } \\ \text { (fórmula de Dahlberg) }\end{array} \\ & \text { fase I } & 0,0053 & \\ \text { A } & \text { fase II } & 0,0050 & 0,0057 \\ & \text { fase III } & 0,0066 & \\ & \text { fase IV } & 0,0059 & \\ & \text { fase I } & 0,0061 & 0,0067 \\ & \text { fase II } & 0,0061 & \\ & \text { fase III } & 0,0079 & \end{array}$

TABELA 3 - Comparações múltiplas de Bonferroni para a região "A".

\begin{tabular}{ccccc} 
fases & $\begin{array}{c}\text { diferença } \\
\text { média }\end{array}$ & $\begin{array}{c}\text { erro } \\
\text { padrão }\end{array}$ & valor t & p \\
I - II & 1,16 & 0,14 & 8,34 & $<\mathbf{0 , 0 0 0 1}$ \\
I - III & 0,91 & 0,14 & 6,34 & $<\mathbf{0 , 0 0 0 1}$ \\
I - IV & 0,55 & 0,15 & 3,57 & $\mathbf{0 , 0 1 6 9}$ \\
II - III & $-0,25$ & 0,05 & $-5,51$ & $\mathbf{0 , 0 0 0 4}$ \\
II - IV & $-0,61$ & 0,10 & $-6,41$ & $<\mathbf{0 , 0 0 0 1}$ \\
III - IV & $-0,36$ & 0,08 & $-4,56$ & $\mathbf{0 , 0 0 2 3}$ \\
\hline
\end{tabular}

não-significativo estatisticamente. Calculando-se a correlação intraclasse, que é uma medida de concordância para o erro sistemático, não foram encontradas concordâncias inferiores a $98 \%$.

Pela tabela 2, observa-se que todas as fases foram, em média, diferentes entre si em relação à densidade óptica, sendo que na fase I encontrouse a maior densidade óptica média e na fase II a menor. Notou-se ainda que, a partir da fase II, a densidade óptica média aumentou. Pelas tabelas 3 e 4, observa-se que a densidade óptica média foi diferente entre quase todas as fases - com exceção da diferença entre as fases I e IV para a região "B", ou seja, a densidade óptica média diminuiu imediatamente após a cirurgia e foi se aproximando do valor inicial com o passar do tempo, sendo que na fase IV já tinha praticamente atingido o valor médio inicial.

TABELA 2 - Médias e desvios-padrão da densidade óptica.

$\begin{array}{ccccc}\text { região } & \text { fase } & \text { média } & \text { d.p. } & \text { n } \\ & \text { I } & 1,80 & 0,52 & 16 \\ \text { A } & \text { II } & 0,64 & 0,16 & 16 \\ & \text { III } & 0,89 & 0,17 & 16 \\ & \text { IV } & 1,25 & 0,30 & 16 \\ \text { B } & \text { I } & 2,97 & 0,69 & 16 \\ & \text { II } & 1,16 & 0,42 & 16 \\ & \text { III } & 1,87 & 0,65 & 16 \\ & \text { IV } & 2,70 & 0,76 & 16\end{array}$

TABELA 4 - Comparações múltiplas de Bonferroni para a região "B".

\begin{tabular}{ccccc} 
fases & $\begin{array}{c}\text { diferença } \\
\text { média }\end{array}$ & $\begin{array}{c}\text { erro } \\
\text { padrão }\end{array}$ & valor t & p \\
I - II & 1,81 & 0,17 & 10,44 & $<\mathbf{0 , 0 0 0 1}$ \\
I - III & 1,10 & 0,17 & 6,62 & $<\mathbf{0 , 0 0 0 1}$ \\
I - IV & 0,27 & 0,10 & 2,62 & 0,1152 \\
II - III & $-0,71$ & 0,12 & $-5,80$ & $\mathbf{0 , 0 0 0 2}$ \\
II - IV & $-1,54$ & 0,20 & $-7,84$ & $<\mathbf{0 , 0 0 0 1}$ \\
III - IV & $-0,83$ & 0,17 & $-4,99$ & $\mathbf{0 , 0 0 1 0}$ \\
\hline
\end{tabular}




\section{DISCUSSÃO}

O tratamento da atresia maxilar, quando diagnosticada e tratada em pacientes jovens, consiste de procedimentos ortopédico-ortodônticos. Muitos estudos enfatizam que a ERMAC é realizada quando há término do fechamento da sutura palatina mediana em pacientes adultos. $\mathrm{O}$ uso de radiografias oclusais maxilares é adequado para se obter uma avaliação da neoformação óssea na separação da sutura palatina mediana $a^{5,12}$.

De acordo com Krebs ${ }^{6}$, $\mathrm{Haas}^{5}$, Phillips et al. ${ }^{11}$ e $\mathrm{Melo}^{7}$, a separação anteroposterior da sutura palatina mediana é em forma de "V", com maior magnitude na região anterior do que na posterior. Notou-se, nesse estudo, que, na maioria dos casos, a separação da sutura foi em forma de "V" com diastema maior entre os incisivos centrais.

A análise da densidade óptica utilizando-se uma escala de alumínio é bem descrita na literatura $^{13,14,16}$ e constitui um método seguro para se monitorar a neoformação óssea ${ }^{3}$.

A escolha das áreas selecionadas para o presente estudo, denominadas áreas "A" e "B", foi baseada em estudos da literatura e visou verificar o padrão de fechamento ósseo da sutura palatina. A região "A" - entre os incisivos centrais, na região da sutura palatina mediana - foi selecionada para observarse o comportamento da densidade óptica por ser uma região importante para a estabilização cirúrgica. A região "B" foi selecionada mais para posterior para permitir o distanciamento do aparelho expansor. Estudos consultados na literatura sobre a densidade óptica na expansão rápida da maxila ressaltam que, logo após o procedimento, os valores da densidade óptica diminuem, sendo que há uma tendência de retorno aos valores iniciais após essa expansão $0^{4,7,15,16}$.

A tabela 1, referente ao erro do método, evidenciou que não ocorreu qualquer evento que caracterizasse um erro sistemático. O erro casual calculado foi pequeno, na ordem da terceira casa decimal, e não comprometeu os resultados obtidos.

No presente estudo, detectou-se a diminuição da densidade óptica na fase II (Tab. 2), pois o valor da densidade óptica média da região "A" diminuiu de $1,80 \mathrm{~mm} / \mathrm{eq} / \mathrm{Al}$ para $0,64 \mathrm{~mm} / \mathrm{eq} / \mathrm{Al}$ e na região "B" houve a diminuição de $2,97 \mathrm{~mm} / \mathrm{eq} / \mathrm{Al}$ para $1,16 \mathrm{~mm} / \mathrm{eq} / \mathrm{Al}$. Aos três meses após o fechamento do parafuso expansor (fase III), esses valores aumentaram (Tab. 2), pois na região "A" o valor da densidade óptica média foi para $0,89 \mathrm{~mm} / \mathrm{eq} / \mathrm{Al}$ e na região "B" foi para $1,87 \mathrm{~mm} / \mathrm{eq} / \mathrm{Al}$. Essa diminuição e posterior aumento da densidade óptica con-

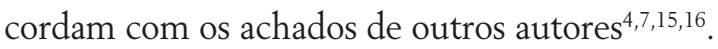

O trabalho de $\mathrm{Melo}^{7}$ encontrou uma equivalência dos valores de densidade óptica aos três meses e dos valores iniciais na dentadura permanente com o tratamento da expansão rápida da maxila. Vasconcelos et al. ${ }^{17}$ observaram, em seu estudo sobre a ERMAC, que os valores finais da densidade óptica após 120 dias de contenção fixa foram significativamente inferiores em relação aos valores iniciais.

Após seis meses do procedimento cirúrgico, observou-se, no presente estudo, que os valores da densidade óptica na região "B" retornaram aos valores iniciais - podendo-se observar na tabela 4 que, ao se comparar as fases I e IV, obteve-se um valor de $p=0,1152$ ( $p>0,05)$, o qual não foi estatisticamente significativo. Já na região "A", não foram alcançados os valores pré-tratamento - podendo-se observar na tabela 3 que todas as fases são, em média, diferentes entre si, inclusive quando comparadas as fases I e IV, com significância estatística $(p<0,05)$. Foram obtidos os seguintes valores médios (em mm/eq/Al) para a densidade óptica (Tab. 2):

- na região "A" $=1,80$ na fase I, 0,64 na fase II, 0,89 na fase III e 1,25 na fase IV.

- na região "B": 2,97 na fase I, 1,16 na fase II, 1,87 na fase III e 2,70 na fase IV.

\section{CONCLUSÕES}

De acordo com os resultados obtidos, pode-se concluir que:

1) Imediatamente após o fim da ativação do parafuso expansor, observou-se valor decrescente 
para as densidades ópticas nas regiões "A" e "B".

2) Após três meses do fim da ativação do parafuso expansor, as densidades ópticas aumentaram nas regiões "A" e "B".

3) Após seis meses do procedimento cirúrgico (ERMAC), as densidades ópticas aumentaram em relação à fase anterior. Na região "A", observou-se que os valores das densidades ópticas não retornaram aos valores pré-tratamento, ou seja, antes da Expansão Rápida da Maxila Assistida Cirurgicamente (ERMAC). Já os valores das densidades óp- ticas médias da região "B" retornaram aos valores iniciais, antes da ERMAC.

\title{
Evaluation of the midpalatal suture optical density six months after surgically assisted rapid maxillary expansion
}

\begin{abstract}
Aim: This study evaluated the new bone formation in the midpalatal suture after surgically assisted rapid maxillary expansion by optical density analysis. Methods: The sample was comprised by 16 patients (six males and ten females). All patients presented maxillary atresia with posterior crossbite and were submitted to SARME. Maxillary occlusal radiographs were taken at four stages (before SARME, immediately after SARME, three months after SARME and six months after SARME). An aluminium scale (step wedge) with eight steps varying from $1 \mathrm{~mm}$ to $8 \mathrm{~mm}$ was adapted at the end of the films. Optical density analysis was performed after digitization of radiographs using the Image Tool ${ }^{\mathrm{TM}}$ software (UTHSCSA, San Antonio, Texas, USA). Region A measured $8 \times 1 \mathrm{~mm}^{2}$ and was located $1.2 \mathrm{~cm}$ from the tangent to the maxillary central incisors at the region of the midpalatal suture. Region $B$ measured $5 \times 9 \mathrm{~mm}^{2}$ and was located $4.3 \mathrm{~cm}$ from the tangent to the maxillary incisors at the region to the midpalatal suture. Results: Statistical analysis of region A revealed statistically significant differences between phases I and II $(p<$ $0.0001)$, and phases I and III ( $p<0.0001)$; statistically significant differences between phases I and IV $(p=0.0169)$, and phases II and III ( $p=0.0004)$; statistically significant differences between phases II and IV ( $<<0.0001)$, and phases III and IV $(p=0.0023)$. In region $B$, there was statistically significant differences between phases I and II ( $p$ $<0,0001)$, and phases II and III ( $p<0.0001)$; no statistically significant differences between phases I and IV ( $p=$ $0.1152)$; in phases II and III ( $p=0.0002)$, phases II and IV ( $<0.0001)$ and phases III and IV ( $p=0.0010)$ statistically significant differences were observed. Conclusions: Optical density analysis improves post-treatment control of SARME procedures by surgeons and orthodontists. After six months, new bone formation at the midpalatal suture is not complete in region " $A$ " $(p<0.05)$, but in region" $B$ " no statistically significant differences were observed ( $p$ $>0.05$ ) in phases I and IV.
\end{abstract}

Keywords: Radiograph. Optical density. New bone formation. Rapid maxillary expansion. 


\section{REFERÊNCIAS}

1. ALTMAN, D. G. Practical statistics for medical research. 1st ed. London: Chapman and Hall, 1991.

2. BAYS, R. A.; GRECO, J. M. Surgically assisted rapid palatal expansion: An outpatient technique with long-term stability. J. Oral Maxillofac. Surg., Philadelphia, v. 50, no. 2, p. 110-113, Feb. 1992

3. BODNER, L. et al. Extraction site healing in rats. Oral Surg. Oral Med. Oral Pathol., St. Louis, v. 75, no. 3, p. 367-371, 1993.

4. COBO, J. M.; VIJANDE, M.; QUINTANILLA, D. S. Evaluation of maxillary disjunction with bone densitometry. J. Clin. Orthod., Boulder, v. 26, no. 2, p. 107-110, 1992.

5. HAAS, A. J. Rapid expansion of the maxillary dental arch and nasal cavity by opening the midpalatal suture. Angle Orthod. Appleton, v. 31, no. 2, p. 73-90, 1961

6. KREBS, A. Expansion of the midpalatal suture studied by means of metallic implants. Acta Odontol. Scand., London, v. 17 , no. 4 , p. $491-501,1959$

7. MELO, M. F. B. Avaliação da densidade óptica da sutura palatina mediana por meio do sistema de radiografia digital por placa fotoativada em pacientes submetidos à disjunção palatina. 2003. Dissertação (Mestrado)-Faculdade de Odontologia, Universidade de São Paulo, São Paulo, 2003

8. MOL, A. Image processing tools for dental applications. Dent. Clin. North Am., Philadelphia, v. 44, no. 2, p. 299-318, 2000

9. NETER, J. et al. Applied linear statistical models. 4th ed. llinois: Irwin Professional Pub, 1996.

10. NORTHWAY, W. M.; MEADE JR., J. B. Surgically assisted rapid maxillary expansion: A comparison of technique, response and stability. Angle Orthod., Appleton, v. 67, no. 4, p. 309-320, 1997
11. PHILLIPS, C. et al. Stability of surgical maxillary expansion in adults. Int. J. Adult Orthodon. Orthognath. Surg., Chicago, v. 7, no. 3, p. 139-146, 1992

12. POGREL, M. A. Surgically assisted rapid expansion in adults. Int. J. Adult Orthodon. Orthognath. Surg., Chicago, v. 7, p. 37-41, 1992.

13. PUPPIN, A. A. C. et al. Análise da densidade óssea pela leitura óptica na região de molares em mandíbulas secas. Rev. Odonto Ciênc., Porto Alegre, v. 13, n. 25, p. 151-165, jun. 1998.

14. SCARPARO, H. C.; COSTA, N. P.; SOUZA, A. C. Apresentação de uma metodologia exploratória para a análise de densidade óssea da mandíbula. Rev. Odonto Ciênc., Porto Alegre, v. 10, n. 20, p. 29-45, dez. 1995.

15. SIMÕES, F. X. P. C.; ARAÚJO, T. M.; BITTENCOURT, M. A. V. Avaliação da maturação óssea na sutura palatina mediana após expansão rápida por meio da imagem digitalizada. R. Dental Press Ortodon. Ortop. Facial, Maringá, v. 8, n. 1, p. 59-67, jan./fev. 2003.

16. VARDIMON, A. D. Rapid palatal expansion. Part 1: Mineralization pattern of the midpalatal suture in cats. Am. J. Orthod. Dentofacial Orthop., St. Louis, v. 113, no. 4, p. 371-378, Apr. 1998.

17. VASCONCELOS, M. H. F. et al. Avaliação da remodelação óssea da sutura palatina mediana com radiografia digitalizada após expansão rápida da maxila assistida cirurgicamente. In: CONGRESSO DA ASSOCIAÇÃO BRASILEIRA DE ORTODONTIA E ORTOPEDIA FACIAL, 5., 2005, Salvador. Anais.. Salvador: Associação Brasileira de Ortodontia e Ortopedia Facial, 2005. p. 1-14
Endereço para correspondência

Eduardo Kazuo Sannomiya

Av. Lacerda Franco 1180 - Aclimação

CEP: 01.536-000 - São Paulo / SP

E-mail: eduardosannomiya@hotmail.com 